\title{
Urgences
}

\section{L'indien de l'Islet au Massacre}

\section{Mario Cotté}

Numéro 8, 4e trimestre 1983

Littérature jeunesse

URI : https://id.erudit.org/iderudit/025121ar

DOI : https://doi.org/10.7202/025121ar

Aller au sommaire du numéro

Éditeur(s)

Urgences

ISSN

0226-9554 (imprimé)

1927-3924 (numérique)

Découvrir la revue

Citer ce document

Cotté, M. (1983). L'indien de l'Islet au Massacre. Urgences, (8), 61-71. https://doi.org/10.7202/025121ar

Ce document est protégé par la loi sur le droit d'auteur. L'utilisation des services d'Érudit (y compris la reproduction) est assujettie à sa politique d'utilisation que vous pouvez consulter en ligne.

https://apropos.erudit.org/fr/usagers/politique-dutilisation/
Cet article est diffusé et préservé par Érudit.

Érudit est un consortium interuniversitaire sans but lucratif composé de l’Université de Montréal, l'Université Laval et l'Université du Québec à Montréal. Il a pour mission la promotion et la valorisation de la recherche. https://www.erudit.org/fr/ 


\section{MARIO COTTÉ}

\section{L'indien de l'Islet au Massacre}




\section{L'INDIEN DE L'ISLET AU MASSACRE}

Appuyé contre la coque de son canot renversé, les yeux miclos, Oeil d'airain contemplait, en silence, la ligne d'horizon nordouest. La grande île disparaissait sous les dernières flammes du somptueux déclin de ce jour d'été. Pas un seul frisson ne venait dérider son visage de terre cuite; un visage que le soleil, le vent et l'air salin avaient tanné comme le cui d'un bouclier.

Oui, figé comme une statue de bronze, Oeil d'airain fixait la ligne $d$ 'horizon, mais ses yeux ne voyaient rien de la dernière flambée du jour. Son esprit avait sombré dans une profonde songerie. Les souvenirs se bousculaient dans sa tête.

\section{Oeil d'airain était vieux.}

II dépoussiérait des souvenirs presque aussi vieux que lui. La lumière de son esprit, bien que vacillante à certains moments, savait toujours éclairer d'une intensité toute naturelle les épisodes qui avaient jalonné son existence. Ceux qui les jalonnaient toujours et ceux qui les jalonneraient tant qu'un souffle de vie I'animerait. Ce soir-là, particulièrement, la lumière avait jailli pour embraser, une à une, les cellules vivantes de son cerveau, dans un ordre que le Grand Manitou avait pu établir. II revivait chaque étape de sa vie avec une rigueur que seule, la veille de son déclin pouvait ensoleiller. II le sentait ce déclin, il s'y soumettait avec cette fatalité que les êtres, en perpétuel contact avec la nature, éprouvent et acceptent comme dénouement inhérent à la naissance.

De plus, au contact de cette nature, vivant continuellement dans le confrontement du quotidien; à la mesure des intempéries, des marées, des étés accablants et des hivers rigoureux, il ignorait tout des accessoires qui allègent et embrouillent la vie de I'homme moderne. Son existence entière fut axée sur le conditionnement que lui imposaient les lois physiques et sociales de sa race et de sa tribu. 
Comme le soleil s'enfonçait dans les eaux, projetant une dernière lueur dans le firmament, Oeil d'airain tressaillit. Une image d'horreur, traumatisante et lancinante passa en arrière-plan sur l'écran de sa vie. Un passage vivide de son enfance. Malgré les soubresauts de son coeur, il ne broncha point. II se laissa absorber de nouveau.

II devait avoir une dizaine d'années à l'époque. Plusieurs membre de sa tribu avaient été pris en chasse par des guerriers d'une tribu rivale. Parmi les siens se trouvaient des femmes et, des enfants comme lui. Les hommes de son groupe avaient avironé jusqu'à l'épuisement.

Par bonheur, ils étaient arrivés à la hauteur de ce havre magnifique dont l'accès était gardé par deux islets massifs et rondouillards. En hâte, les canots furent tirés sur la grève, et comme certains parmi les Indiens se rappelaient vaguement l'existence d'une grotte, tout le monde se mit à sa recherche. Désespérés mais lucides, ils ne prirent pas de temps à en repérer l'entrée. Quelques arbres la masquaient, cependant, munis de leurs haches, les hommes s'employèrent à les abattre et à barricader la cache comme ils le purent. Tout le monde disparut à I'intérieur et attendit. On avait entassé au fond de la caverne les quelques victuailles que les canots avaient transportées en plus des fugitifs.

Deux ou trois braves s'empressèrent d'aller dissimuler les embarcations dans les bois environnants. Une certaine appréhension habitait les âmes; la tension montait à la mesure de l'attente. Toutefois les Indiens, et Oeil d'airain s'en est toujours souvenu, parvenaient à contenir leurs émotions et leur visage restait de marbre.

Oeil d'airain poussa un long soupir. Une à une, les étoiles 
s'accrochaient à la voûte céleste dans un ballet de dorures lumineuses. La nuit étendait peu à peu sur ce monde resplendissant de richesses primitives sa cape de velours noir. Après un dernier regard jeté sur les perles argentées que la vague venait dissoudre sur le sable de la berge, Oeil d'airain vint s'asseoir à l'entrée de la caverne. Croisant les jambes et $\mathbf{s}$ adossant à un tuf que les grandes marées avaient souvent poli, il retourna à ses réflexions.

La sentinelle qui faisait le guet scrutait sans cesse le large. Toujours rien à signaler. Rien que le calme lourd et revêche. Une quiétude par trop obsédante.

Or, dans l'obscurité, plusieurs canots glissaient sur l'eau, mollement commandés par des fantômes faisant du sautemouton avec les nuages. Les guerriers qu'ils transportaient avaient déposé leurs avirons à côté d'eux. Allongés à plat ventre au fond des embarcations, ils avaient passé par-dessus bord soit leur bras gauche, soit leur bras droit, et de cette façon, ils pouvaient se diriger, tout en se dissimulant du mieux qu'ils pouvaient. C'est ainsi qu'ils trompèrent la vigilance de la sentinelle.

Ils contournèrent l'islet et accostèrent à son extrémité sudouest. En plusieurs dizaines, ils se faufilèrent, partagés en deux groupes, comme des félins, l'oreille aux aguets, à l'affût du moindre chuchotement de feuille. Rampant plus que marchant, ils s'approchèrent du refuge de leurs victimes, semblables à une meute de loups prête à fondre sur sa proie.

La sentinelle toujours en éveil continuait à fouiller la nuit, étanchément noire. Soudain, une plainte d'animal blessé déchira I'air, suivie d'un râle à peine perceptible.

En moins de deux, des torches s'allumèrent, un rugissement épouvantable fut poussé par toutes les poitrines à l'unisson, dans un seul cri et une course échevelée conduisit les belligérants vers la grotte. Ô malheur, de solides barricades se dressaient dans l'entrée et une pluie de flèches s'abattit sur les attaquants, décimant leurs rangs. La bataille fut engagée au clair de lune qui 
venait de sortir et la poésie de cette nuit préhistorique trouvera des rimes bien sanglantes.

Plusieurs fois, I'assaut fut donné et l'élan brisé sur la palissade qui tenait bon. Des têtes furent fracassées, des visages se figèrent dans d'abominables rictus. Certains boitaient en essayant de retirer une flèche plantée dans la jambe ou dans la cuisse. Après maints échecs, le chef des attaquant eut une idée diabolique. II imita le glapissement du renard et ses hommes cessèrent le combat. Regroupés autour de lui, il leur fit part d'un projet fort simple et fort efficace, qui avait germé dans sa cervelle laborieuse.

Oeil d'airain se souvenait de ce glapissement, de cette clameur qui régnait sur le combat et qui s'était éteinte presque subitement.

De l'intérieur, on cherchait bien à discerner quelque chose, mais les assaillants s'étaient retirés trop loin; même le murmure de leurs voix, qui portent néanmoins à bonne distance la nuit, ne leur parvenait point.

Une demi-douzaine d'entre eux revinrent, une torche à la main et s'approchèrent témérairement de la palissade, dans un geste concerté. De leurs torches, ils mirent le feu à divers endroits. L'incendie se propagea comme un feu de paille. Ce furent cris, gémissements, hurlements. Certains, parmi les assiégés, réussirent à franchir le brasier mais ce fut pour se faire cribler de flèches ou pourfendre d'un coup de tomahawk. La scène fut horrible à voir, mais les vainqueurs, ivre de haine et de sang, ricanaient comme des démons.

Tout à coup surgit un garçonnet. II réussit à franchir la vague de feu. Son petit pantalon avaient une cuissière enflammée. II criait et se démenait, puis se roula dans le sable avant que l'un des guerriers ne le saisisse par un bras. L'enfant, le futur Oeil 
d'airain, se sentit prétrifié à la vue du farouche individu qui le traînait à sa suite. Heureusement ses brûlures superficielles ne le faisaient pas trop souffrir. II avait également de la chance qu'il en soit ainsi, car on le ficela comme un boudin et on le coucha au fond d'un canot, comme un vulgaire ballot de fourrure.

Ce souvenir fut particulièrement cuisant au coeur d'Oeil d'airain. Les traits de son visage s'étaient crispés. II dut prendre le temps de se rasséréner. Quelle humiliation! Lui, le futur guerrier à qui on avait déjà enseigné la fierté, I'honneur et la bravoure. II trépignait, pensait déjà à s'échapper, même à tirer vengeance de cette ignominieuse déroute.

II entendit le pas des guerriers ennemis qui revenaient à leurs canots. II se tranquilisa tout de suite, en jouant la soumission. II ne pouvait supporter les ricanements de ces bourreaux qui en plus d'avoir massacré tous les siens rapportaient, accrochés à leur ceinture de ces glorieux trophées qui consistaient en des scalps fraîchement prélevés sur de malheureux crânes.

Il lui parvenait une odeur de fumée, ignoble à ses narines, et qui se mélangeait à des relents de cadavres grillés et de bois de mer calcinés.

L'enfant ferma les yeux sur une source de larmes prête à jaillir. Les canots à la mer, les hommes se mirent à avironner. Le périple fut très long. On libéra Oeil d'airain de ses liens et il put s'asseoir sur l'un des bancs. De farouches regards le dévisageaient avec rigueur. II comprit ce que cela voulait dire et se tint coi; se contentant de regarder défiler les montagnes, les rivières et les baies qui jalonnaient la rive.

Après plusieurs jours et plusieurs arrêts sur la côte, la flottille atteignit une grande baie où s'élevaient un village, une espèce de bourgade.

Oeil d'airain fut amené par les guerriers jusque sur la place centrale du village. On le montra au grand sorcier qui le trouva fort beau et de belle allure. II fut donc conseillé au grand chef de lui laisser la vie sauve et de l'élever avec les autres petits Indiens. 
On lui enseignerait les habitudes et les coutumes de sa nouvelle tribu.

Oeil d'airain revoit cette bourgade. Elle lui était apparue dans son immensité, avec ses tentes bien alignées, dont quelquesunes, celle du sorcier, du grand chef et celles des chefs intermédiaires, dépassaient les autres par leur dimension. II avait remarqué les crânes séchés, les chevelures suspendues à la porte de plusieurs tentes, en plus des panaches de cerfs, d'élans, et des nombreuses peaux d'ours. Une étoile filante tomba du firmament comme une escarbille sonnant l'arrêt du temps.

Les années passèrent et Oeil d'airain devint un bel adulte. Le plus naturellement et le plus heureusement du monde, il s'éprit secrètement de Perle de rosée, la fille du grand chef de toute la tribu. Ses sentiments furent partagés; secrètement aussi par cette beauté au regard de source limpide. Leur amour ne demandait qu'à jaillir en cascade de sève du plus intime de l'un jusqu'au plus intime de l'autre. Leur jeunesse ne pouvait s'y tromper; non plus que leur coeur, leur âme et leur esprit.

Le destin devait cependant leur asséner un coup fatal. Dès sa naissance, Perle de rosée avait été promise. Oui, promise au fils aîné de l'un des chefs intermédiaires, c'est-à-dire le plus âgé de ces chefs qui prendrait un jour en main les destinées de la tribu, Perle de rosée n'ayant pas de frère. La douleur fut abominable et le chagrin gonfla de silence le coeur d'Oeil d'airain, lorsqu'il fut mis en présence de la toute belle et qu'elle lui fit cette affreuse révélation, un soir, près du tronc d'un érable rouge. II sentit un froid polaire lui courir dans les veines, alors que l'instant d'avant, l'ardeur de la passion ensoleillait sa vie; toute sa vie.

II détacha à jamais son regard de sa bien-aimée. S'enfuit en courant vers la baie et dans une rage folle, sauta dans un canot pour se mettre à avironner comme un forcené. On eut beau le poursuivre, I'auréoler de flèches, vociférer à ses trousses, Oeil d'ai- 
rain fila comme si les serviteurs du Grand Manitou essuent arraché aux flots son canot pour le guider vers les nuages.$$
-\mathrm{II}-
$$

Comme par un enchantement, sans qu'il y fut pour quoi que ce soit, Oeil d'airain se retrouva devant la caverne d'où il avait été enlevé auparavant. Rien n'avait changé. Les islets montaient toujours la garde à l'entrée de la baie, les caps veillaient sans relâche, la grande île, au large, semblait toujours ensommeillée.

Les fortes marées avaient nettoyé la plage. Plus un seul ossement, sauf à l'intérieur de la caverne. II ramassa ces restes que les charognards avaient oubliés, les plaça dans son canot et s'en fut les ensevelir sur la terre ferme, près du cap, afin que le repos de ces braves fut assuré.

II s'était peu à peu habitué à cette nouvelle existence. Son beau carquois fait de peau de bête s'était empli de flêches façonnées avec doigté. II harponnait le saumon de la rivière qui se jetait dans la baie par le sud-ouest. Habile chasseur, il se nourrissait de ce gibier de choix qui pullulait dans son domaine et dans les alentours. Etant donné qu'il n'avait à satisfaire que ses besoins essentiels, peu nombreux d'ailleurs, le reste du temps, il le pasait à flâner. Comme tous les Indiens, il jouissait d'une âme de poète, aussi aimait-il escalader les sites environnants d'où la vue plongeait sur le grand fleuve, les forêts, les bosquets et les horizons à perte de vue.

La douleur d'avoir perdu Perle de rosée s'estompait avec les années. Seule sa douce image venait créer en lui des moments de sérénité de plus en plus nombreux. Cette présence imaginaire le comblait tout autant que s'il avait eu une compagne à ses côtés.

Depuis des lustres, il vivait ainsi, en sédentaire. II s'était même laissé aller à sculpter divers objets, ayant constaté que les bois de mer, très secs, se laissaient plus facilement tailler.

Or, un beau matin, alors qu'il en était à fabriquer un nouvel 
aviron, Oeil d'airain eut la surprise de sa vie. II en resta bouche bée, croyant rêver. II se mit à contempler la scène avec une curiosité qu'il ne s'était jamais connue.

Glissant majestueusement sur les flots bleu azur du fleuve, faisant voile en direction de la baie, trois caravelles voguaient... à la file indienne.

II eut beau écarquiller les yeux, se les frotter, les écarquiller de nouveau, c'était bien cela. Trois gigantesques embarcations, munies de mâts et de grands drapeaux gonflés par la brise fraîche, semblaient se diriger droit sur son repère.

II attendit.

Par la suite, un canot fut lancé vers le rivage, monté par quelques hommes qu'Oeil d'airain n'arrivait pas à distinguer. A mesure que le canot approchait, il distingua bien deux Indiens et trois autres individus drôlement affublés de vêtements bizarres. Cependant, ce qui médusait Oeil d'airain, c'est que ces trois autres hommes étaient des visages pâles. II n'avait jamais vu cela et ne broncha pas d'un millimètre de son poste d'observation.

Le canot accosta enfin. Les deux Indiens débarquèrent suivis de I'un des blancs dans son drôle d'acoutrement et d'un autre homme, très imposant, à la barbe forte, portant épée au côté gauche et une singulière coiffe qui n'avait rien des plumes que portent les chefs indiens.

Oeil d'airain s'avança avec précaution. L'homme à la barbe drue s'approcha de lui et, grâce à un interprète, lui fit comprendre qui il était, en s'inclinant tout d'abord devant Oeil d'airain qui n'en croyait pas ses yeux.

Jacques Cartier (car c'était bien lui) offrit des présents et Oeil d'airain lui rendit la pareille en lui offrant des peaux de bête et une paire de mocassins.

C'est le moment où Oeil d'airain raconta à son illustre visiteur I'histoire de la tragédie qui avait empoisonné son existence. L'un 
des compagnons de Jacques Cartier notait toutes les péripéties du massacre sur un immense rouleau de papier, ce qui étonna encore Oeil d'airain. Mais il était intelligent et comprit que I'homme faisait des signes qui voulaient dire quelque chose.

Une fois le récit terminé et de nouvelles civilités échangées, Jacques Cartier pria son hôte de lui laisser visiter les environs, avec promesse de ne rien dérober ni endommager. Là-dessus, l'étrange homme blanc remit sa coiffure, de même que ses hommes et tous s'inclinèrent avec courtoisie et se retirèrent. Oeil d'airain ne put faire autre que de remarquer leurs bottes, luisantes, de couleur brune, avec des boucles dessus.

Il les suivit à distance à bord de son canot, les vit accoster ailleurs, dans la baie, retourner à leur caravelles, dont les voiles se gonflèrent bientôt, et prendre la direction du large tout en suivant le littoral de la grande île.

Ainsi, de par les récits de voyage de Jacques Cartier, avonsnous appris officiellement I'histoire de ce carnage. Néanmoins, la légende sera la plus forte et la plus convaincante des deux et nous le verrons tout à I'heure.

Oeil d'airain chassait et pêchait toujours. Malgré le poids des ans, son pas était toujours alerte et sa force physique ne semblait pas décidée de l'abandonner encore. De temps à autre, dans le cours de son sommeil, ou encore lorsqu'il s'arrêtait à scruter les profondeurs de l'eau, le visage de Perle de rosée lui apparaissait sous la forme de traits si purs et si harmonieux qu'il en était tout bouleversé, croyant qu'elle allait lui parler et lui offrir du maïs.

Oeil d'airain se retira dans sa caverne. En attisant ainsi ses souvenirs, il avait fait en quelque sorte son examen de conscience. Stoïquement, il tira une dernière bouffée de son calumet. II dispersa les quelques cendres encore chaudes du feu de rondins qu'il avait allumé plus tôt. Depuis quelque temps, il sentait la mort toute prête à le cueillir. Par une sorte de divination 
mystérieuse, il avait entendu son appel.

Quelques lunes plus tard, il sut que le grand moment du passage de vie à trépas était au seuil de sa caverne. II plaça son carquois à gauche de sa couche, son arc et son tomahawk à droite. A ses pieds, ses mocassins et son plumet à la tête; puis il sortit jeter un dernier coup d'oeil dans les environs. Son dernier regard fut pour le cap qui n'était pas encore Enragé. II prononça quelques mots dans son idiome, regagna son repaire et se coucha.

II fit la paix avec sa propre vie. Sur la voûte sombre de la caverne qui avait tant de fois abrité ses réflexions, ses repas, son sommeil et ses rêves, il voyait se dessiner l'antichambre de la mort. II sentit le poids de l'engourdissement se saisir de son être. II ferma les yeux et peu à peu la vie se retira de son corps. En à mesure qu'elle régressait ainsi, Oeil d'airain s'allégeait de plus en plus.

Tout à coup, sous son regard ébloui, il vit s'ouvrir devant lui la porte d'un grand jardin. C'était le territoire des chasses éternelles. Oeil d'airain y pénétra et s'en fut prendre possession de son nouveau domaine.

Quelquefois, son âme revient hanter les abords de l'Islet au Massacre. Pour le visiteur solitaire, il adopte la forme d'un revenant splendide. Superbe guerrier trasısparent, d'une voix gutturale, il relate l'événement qui a marqué, non seulement son enfance, mais toute sa vie. Sans omettre un seul épisode, avec une grande noblesse et une fière dignité, il vous fixe de son regard d'aigle. Son récit terminé, le fantôme se dissipe comme la rosée fond au soleil de l'aube. Alors que cette histoire prête à interprétation, la légende, grâce au miracle de l'émerveillement, vivra éternellement. 